\title{
Short-time diffusion of charge-stabilized colloidal particles: Generic features
}

\author{
Marco Heinen, ${ }^{a}$ Peter Holmqvist ${ }^{a}$ Adolfo J. Banchio ${ }^{b}$ And \\ Gerhard NÄGELE ${ }^{a *}$ \\ ${ }^{a}$ Institut für Festkörperforschung, Forschungszentrum Jülich, D-52425 Jülich, \\ Germany, and ${ }^{b} F a M A F$, Universidad Nacional de Córdoba, IFEG-CONICET \\ Ciudad Universitaria, 5000 Córdoba, Argentina.E-mail: g.naegele@fz-juelich.de
} (Received April 20, 2010; accepted July 10, 2010)

\begin{abstract}
Analytic theory and Stokesian dynamics simulations are used in conjunction with dynamic light scattering, to investigate the role of hydrodynamic interactions in the short-time diffusion in suspensions of charge-stabilized colloidal particles. The particles are modeled as solvent-impermeable charged spheres, repelling each other via a screened Coulomb potential. Numerical results for self-diffusion and sedimentation coefficients, and hydrodynamic and short-time diffusion functions are compared to experimental data, for a wide range of volume fractions. The theoretical predictions for the generic behavior of short-time properties obtained from this model are shown to be in full accord with our experimental data. In addition, the effects of microion kinetics, non-zero particle porosity, and residual attractive forces on the form of the hydrodynamic function are estimated. This serves to rule out possible causes for the strikingly small hydrodynamic function values determined in certain synchrotron radiation experiments.
\end{abstract}

PREPRINT: Journal of Applied Crystallography

A Journal of the International Union of Crystallography 


\section{Introduction}

Dispersions of charged colloidal particles undergoing correlated Brownian motion form a particularly important class of soft matter systems ubiquitously encountered in chemical industry, food science and biology (Pusey, 1991; Nägele, 1996; Bowen \& Mongruel, 1998; Retailleau et al., 1999; Bowen et al., 2000; Riese et al., 2000; Koenderink et al., 2003; Gapinski et al., 2005; Prinsen \& Odijk, 2007). The calculation of diffusion transport properties for these systems is challenging since one needs to cope, in addition to direct electro-steric and van der Waals inter-particle forces, with the solvent-mediated hydrodynamic interactions (HIs). The latter type of interaction is long-ranged and non-pairwise additive in non-dilute systems.

In the present paper, using analytic theory, simulation and light scattering, we discuss generic features of diffusion in fluid-like ordered suspensions of charge-stabilized colloidal spheres, as observed on a short-time colloidal scale. The most frequently used experimental methods to study the dynamics of charge-stabilized systems are dynamic light scattering (DLS) and X-ray photon correlation spectroscopy (XPCS). In both methods, the dynamic structure factor, $S(q, t)$, is determined as a function of scattering wavenumber $q$ and correlation time $t$. At short times, $S(q, t)$ decays exponentially according to (Pusey, 1991)

$$
S(q, t) \propto \exp \left\{-q^{2} D(q) t\right\}
$$

The short-time diffusion function (Nägele, 1996),

$$
D(q)=d_{0} H(q) / S(q)
$$

is determined by the ratio of the positive definite hydrodynamic function, $H(q)$, and the static structure factor $S(q)$. At zero particle concentration, $D(q)$ reduces to the single-particle diffusion coefficient $d_{0}$. The hydrodynamic function, $H(q)$, obtained experimentally by the short-time measurement of $D(q)$ in combination with a static IUCr macros version 2.1.1: 2009/05/11 
scattering experiment determining $S(q)$, quantifies the influence of the HIs on colloidal short-time diffusion and sedimentation. Without HIs, $H(q)$ is a constant function equal to one. Any $q$-dependence of $H(q)$ reflects the influence of HIs. In the limit of large wavenumbers compared to the position, $q_{m}$, of the principal peak of $S(q)$, the function $H(q)$ becomes equal to the normalized short-time self-diffusion coefficient, $d_{S} / d_{0}$, which is smaller than one when HIs are significant. In the limit of $q \rightarrow 0$, $H(q)$ reduces to the sedimentation coefficient $K=U_{s} / U_{0}$. Here, $U_{s}$ is the mean sedimentation velocity of particles in a uniform suspension subject to a weak constant force field, and $U_{0}$ is the single-sphere sedimentation velocity under the same force field. For an arbitrary value of $q, H(q)$ has the meaning of a generalized sedimentation (or mobility) coefficient, linearly relating a spatially periodic force field of wavelength $2 \pi / q$ acting on the particles, to the resulting spatially periodic particle drift velocities. The most significant value of $H(q)$ is its principal peak height, $H\left(q_{m}\right)$, attained at a wavenumber which coincides practically with the position, $q_{m}$, of the principal peak of $S(q)$. The value of $H\left(q_{m}\right)$ relates to the short-time relaxation of density fluctuations of wavelength $2 \pi / q_{m}$, comparable in size to the radius of the dynamic cage of nextneighbor particles formed around each particle.

A theoretical discussion of $H\left(q_{m}\right)$ for charged spheres as function of the particle volume fraction $\phi$ was given recently by Gapinski et al. (2010). Therein, it was shown that, due to the increasing strength of near-field HIs with increasing concentration, $H\left(q_{m}\right)$ behaves non-monotonically in $\phi$ at low salinity, showing an initial increase towards its maximal value larger than one, followed by a decline for further enlarged $\phi$ to values that can be smaller than one. This behavior differs from that of the sedimentation and short-time self-diffusion coefficients, which both decrease monotonically with increasing $\phi$. Gapinski et al. (2010) provided in addition the universal limiting freezing line for $H\left(q_{m}\right)$, which leads to a useful map of hydrodynamic function peak 
values attainable in the fluid regime.

Our calculations of short-time diffusion properties are based on the one-component macroion fluid (OMF) model which describes the colloidal particles as uniformly charged spheres with stick hydrodynamic boundary conditions on their surfaces (Banchio \& Nägele, 2008). The spheres interact by a screened Coulomb potential of DerjaguinLandau-Verwey-Overbeek (DLVO) type. The accuracy of two analytical methods to calculate short-time properties is assessed by comparison with results from numerically accurate, but computationally expensive, accelerated Stokesian Dynamics (ASD) simulations (Banchio \& Brady, 2003; Gapinski et al., 2005; Banchio \& Nägele, 2008), and with DLS data on charged silica spheres in a toluene-ethanol mixture.

The first analytical method is the $\delta \gamma$ scheme by Beenakker \& Mazur (1984). It accounts in an approximate way for many-body HIs contributions, but ignores higherorder near-field HIs contributions and lubrication effects. The $\delta \gamma$ scheme gives qualitatively good results for $H(q)$ throughout the liquid colloid phase. It can be further improved in its prediction of $H(q)$, when its microstructure-independent self part is replaced by an accurate simulation expression (Gapinski et al., 2005; Banchio \& Nägele, 2008).

The second analytical method, referred to as the fully pairwise-additive (full PA) approximation, completely accounts for HIs on the pairwise level, but it ignores threebody and higher-order hydrodynamic contributions. This method is exact for very low values of $\phi$, where HIs are pairwise additive. Therefore, by comparison with ASD simulation data for the considered short-time property, the full PA scheme allows to infer the importance of three-body and higher-order HIs contributions. Different from the $\delta \gamma$ scheme, the full PA method is bound to fail at higher concentrations due to its complete neglect of many-body HIs. Both analytic methods require $S(q)$ or, alternatively, its real-space analogue, the radial distribution function $g(r)$, as the 
only input. The static input is computed using an improved version (Heinen et al., to be submitted) of the computationally very efficient particle background-corrected rescaled mean spherical approximation (PBRMSA) scheme by Snook \& Hayter (1992), discussed further down. The good accuracy of this largely unknown analytical scheme is demonstrated by comparison with Monte-Carlo (MC) simulations, and results from the accurate but numerically far more elaborate Rogers-Young (RY) scheme (Rogers \& Young, 1984).

The theoretical and simulation results for the short-time properties are compared with our (dynamic) light scattering data on low-salinity suspensions of charged silica spheres, and with scattering data on charge-stabilized systems by some other groups. The results presented in this paper serve to highlight generic features of short-time diffusion properties of charged particles, in comparison to those of neutral hard spheres, and to explore the range of applicability of useful analytic expressions describing a non-analytical $\phi$-dependence of $d_{S}, K$ and $H\left(q_{m}\right)$ for small values of $\phi$ and long-range repulsion (low salinity).

In addition, we discuss qualitatively the changes in $H(q)$ when interaction contributions not included in the OMF model are operative. We analyze the effect of short-range attractions caused, e.g., by van der Waals forces, the influence of particle porosity, hydrodynamic screening, and the additional dynamic friction due to the electrokinetic relaxation of the microionic cloud dragged along each colloidal sphere. This discussion serves to scrutinize possible causes for the strikingly small values for $H(q)$, purportedly determined in synchrotron radiation experiments by Robert and collaborators (Robert, 2001; Robert, 2007; Autenrieth et al., 2007; Robert et al., 2008; Grübel et al., 2008), for certain low-salinity systems. These values are considerably smaller than those of neutral hard spheres. The findings by Robert et al. are incompatible not only with short-time predictions based on the OMF model, but also with experimen- 
tal data for all other (low-salinity) charge-stabilized systems we are aware of, where $H\left(q_{m}\right)$ is found to be always larger than the peak value of hard spheres at the same $\phi$. We correct an incorrect statement by Robert (2007) made on the equality of the self-diffusion coefficient for neutral and charged spheres.

While the present work focuses on short-time dynamics, we point here to recent DLS experiments showing that a far-reaching scaling behavior of the dynamic structure factor of neutral hard spheres (Segrè \& Pusey, 1996), relating short-time to long-time dynamics, is approximately valid also for charged colloids (Holmqvist \& Nägele, 2010). Short-time diffusion in charged colloids is not only an interesting topic in its own right. Its knowledge is also a prerequisite for a better understanding of the long-time dynamics.

\section{Methods of calculation and experimental details}

The presented analytic and computer simulation results for the short-time diffusion properties and $S(q)$, are based on the OMF model. In this simplifying model, a colloidal sphere and its cloud of neutralizing microions are described as a uniformly charged sphere of diameter $\sigma$, interacting electrostatically by an effective pair potential, $u(r)$, of DLVO type (Nägele, 1996)

$$
\frac{u(r)}{k_{B} T}=L_{B} Z^{2}\left(\frac{e^{\kappa a}}{1+\kappa a}\right)^{2} \frac{e^{-\kappa r}}{r}, \quad r>\sigma=2 a
$$

The screening parameter, $\kappa$, is given by

$$
\kappa^{2}=\frac{4 \pi L_{B}\left[n|Z|+2 n_{s}\right]}{1-\phi},
$$

where $n$ is the colloid number density, $n_{s}$ is the number density of added 1-1 electrolyte, and $\phi=(4 \pi / 3) n a^{3}$ is the colloid volume fraction of spheres with radius $a$. Furthermore, $Z$ is the effective particle charge in units of the elementary charge $e$, IUCr macros version 2.1.1: 2009/05/11 
and $L_{B}=e^{2} /\left(\epsilon k_{B} T\right)$ is the Bjerrum length of the suspending Newtonian solvent of dielectric constant $\epsilon$ at temperature $T$. The factor $1 /(1-\phi)$ is frequently introduced to correct for the free volume accessible to the microions. Hydrodynamically, the colloidal particles are treated as non-permeable rigid spheres with stick boundary conditions on their surfaces. The OMF model captures essential features of charge-stabilized suspensions, for systems where the short-range van der Waals forces can be neglected.

We use two efficient analytical methods to calculate $H(q)$ and its small- and large$q$ limiting values $K$ and $d_{S} / d_{0}$. The first method is the zeroth-order $\delta \gamma$ method by Beenakker \& Mazur (1984) which invokes a partial re-summation of many-body HIs contributions. It uses truncated hydrodynamic mobility tensors without lubrication corrections. An extensive comparison (Gapinski et al., 2005; Banchio \& Nägele, 2008; Banchio et al., 2006; Gapinski et al., 2007; Gapinski et al., 2009) of the $\delta \gamma$ scheme predictions for $H(q)$ with ASD simulations results has shown that it reproduces the $H(q)$ of charge-stabilized particles quite well, to a degree even better than for neutral hard spheres where lubrication is strong, when in place of the original self-part the accurate ASD simulation result for $d_{S} / d_{0}$ is used so that $H(q)=d_{S}^{\mathrm{ASD}} / d_{0}+H_{d}^{\delta \gamma}(q)$. At smaller $\phi$, one can more conveniently use the full PA scheme result for $d_{S}$. This improvement can be understood from noting that $d_{S}$ is underestimated by the $\delta \gamma$ scheme, since its zeroth-order expression for $d_{S}$ used here does not account for the pair structure of charged spheres. However, and most importantly, the wavenumberdependent distinct part, $H_{d}(q)$, is well described by the $\delta \gamma$ scheme (Gapinski et al., 2005; Banchio \& Nägele, 2008). In the following section, we exemplify the accuracy of the self-part corrected $\delta \gamma$ scheme, in comparison to MC simulations and experimental results for $H(q)$, for the most interesting case of suspensions of strongly correlated particles at lower salt content.

The second analytical method is the fully pairwise additive (full PA) approximation.

IUCr macros version 2.1.1: 2009/05/11 
It uses tables of numerically precise values for the two-body mobility tensors provided by Jeffrey \& Onishi (1984), Kim \& Mifflin (1985), and Jones \& Schmitz (1988). The full PA scheme is a significant improvement over earlier two-body approximations for $H(q)$, also commonly referred to as PA schemes, where only a long-distance form of the hydrodynamic pair mobilities in terms of a $(a / r)$ inverse pair distance expansion truncated after a few terms has been used (Nägele et al., 1994; Nägele et al., 1995; Nägele, 1996; Watzlawek \& Nägele, 1999; Robert, 2001; Robert, 2007). The full PA method becomes exact at very small $\phi$ where the HIs are truly pairwise additive. However, it necessarily fails at larger $\phi$ since it disregards three-body and higherorder hydrodynamic contributions.

The static input $S(q)$ required by both analytical methods, is obtained using the computationally highly efficient, particle background-corrected rescaled mean spherical approximation (PBRMSA) by Snook \& Hayter (1992). We have augmented this method by a simple density rescaling of the screening parameter in Eq. (4). This rescaling leads to very accurate structure factors, as shown recently by an extensive comparison with simulation data, and results from the similarly accurate but numerically far more costly Rogers-Young integral equation scheme (Heinen et al., to be submitted). Two examples demonstrating the accuracy of the PBRMSA-S $(q)$ are discussed in the following section.

Furthermore, we calculate $H(q)$ using the accelerated Stokesian dynamics (ASD) simulation code by Banchio \& Brady (2003), extended to the OMF model of charged spheres (Gapinski et al., 2005; Banchio et al., 2006; Banchio \& Nägele, 2008). This elaborate simulation method accounts for many-body HIs and lubrication. The calculated hydrodynamic function exhibits a pronounced $O\left(N^{-1 / 3}\right)$ dependence on the number, $N$, of particles in the basic simulation box. A finite-size scaling extrapolation procedure originally used by Ladd (1990) for neutral spheres, is applied to 
extrapolate to the $H(q)$ of a macroscopically large system. The good agreement of the finite-size corrected ASD $H(q)$ with the full PA scheme result at small volume fractions $\left(\phi<10^{-2}\right)$, where the latter scheme becomes exact, demonstrates the validity of Ladd's finite size scaling method also in its application to charged spheres. Stokesian dynamics simulations of $H(q)$ are computationally expensive even when the accelerated code is used. Therefore, fast approximate schemes such as the $\delta \gamma$ method are still on demand, in particular when various system parameters need to be varied to explore generic features.

For dilute, low-salinity systems of strongly charged particles, characterized by $q_{m} \propto$ $\phi^{1 / 3}$, very simple expressions with fractional exponents apply,

$$
\begin{aligned}
K & \approx 1-a_{s} \phi^{1 / 3} \\
d_{S} / d_{0} & \approx 1-a_{t} \phi^{4 / 3} \\
H\left(q_{m}\right) & \approx 1+p_{m} \phi^{1 / 3} .
\end{aligned}
$$

These expressions have been derived using the leading-order far-field forms of the hydrodynamic mobilities (Nägele, 1996; Nägele et al., 1994; Nägele et al., 1995; Watzlawek \& Nägele, 1999). The coefficients $a_{s} \approx 1.6-1.8$ and $a_{t} \approx 2.5-2.9$ in the expressions for $K$ and $d_{S} / d_{0}$ depend to a certain extent on the particle charge and size. The coefficient $p_{m}>0$ related to $H\left(q_{m}\right)$ also depends on $Z$ and $\kappa a$ (Gapinski et al., 2010; Banchio \& Nägele, 2008). All coefficients are typically larger for more structured suspensions, signalled by a higher peak value of $S\left(q_{m}\right)$. As we will show, the $\phi$-interval where the expression for $d_{S}$ in Eq. (6) applies is broader than the interval for the collective properties $K$ and $H\left(q_{m}\right)$.

It will prove useful in what follows to compare the short-time results for charged colloidal spheres with those of neutral hard spheres at the same $\phi$. Cichocki and collaborators derived the following virial expansion results for neutral hard spheres IUCr macros version 2.1.1: 2009/05/11 
(HS) (Cichocki et al., 1999; Cichocki et al., 2002),

$$
\begin{aligned}
K^{\mathrm{HS}} & =1-6.546 \phi+21.918 \phi^{2}+\mathcal{O}\left(\phi^{3}\right) \\
d_{S}^{\mathrm{HS}} / d_{0} & =1-1.832 \phi-0.219 \phi^{2}+\mathcal{O}\left(\phi^{3}\right),
\end{aligned}
$$

valid to quadratic order in $\phi$. These truncated virial results fully account for HIs up to the three-body level.

The hydrodynamic function peak height of hard spheres is given to excellent accuracy by (Banchio et al., 1999)

$$
H^{\mathrm{HS}}\left(q_{m}\right)=1-1.35 \phi
$$

with the linear $\phi$-dependence valid up to the freezing transition concentration.

The short-time experimental data presented in this work have been obtained using dynamic light scattering (DLS) from fluid-ordered and nearly monodisperse suspensions of negatively charged trimethoxysilylpropyl methacrylate (TPM) coated silica spheres (Philipse \& Vrij, 1988), dispersed in an index-matching $80: 20$ toluene-ethanol solvent mixture at $T=20^{\circ} \mathrm{C}$ and $L_{B}=8.64 \mathrm{~nm}$. The particle radius determined by small angle X-ray scattering (SAXS) is $a=136 \mathrm{~nm}$, and the size polydispersity is 0.06 . The salinity of residual $1-1$ electrolyte is below $1 \mu \mathrm{M}$. Monovalent counterions (hydrated protons) are released into the solvent from the coated silica surfaces. The studied charged silica system freezes at $\phi \approx 0.16$ where $S\left(q_{m}\right) \approx 3.2$. The DLS measurements were made using a light scattering set-up by the ALVLaservertriebsgesellschaft (Langen, Germany) and a ALV-5000 multi-tau digital correlator. We carefully checked that there is no noticeable multiple scattering.

\section{Results}

In the following, we present our theoretical and simulation results for charged colloidal particles based on the OMF model, in comparison with our light scattering data on IUCr macros version 2.1.1: 2009/05/11 
coated charged silica spheres, and scattering data by two other groups. The results for charged colloidal spheres (CS) are compared in addition with corresponding findings for neutral hard spheres (HS), to highlight salient differences in the short-time diffusion properties which are largest when low-salinity charge-stabilized systems are considered. Therefore, only charge-stabilized systems of lower salinity are considered. For the influence of added salt on the short-time diffusion coefficients and $H(q)$ we refer to extensive ASD simulations and analytic calculations published in (Gapinski et al., 2009; Gapinski et al., 2010; Banchio \& Nägele, 2008; Banchio et al., 2008). Therein, it is shown that the OMF-based diffusion properties cross over monotonically, with increasing salt concentration, from their zero-salt values to those of neutral hard spheres. This expected behavior is reflected also in the static structure factor.

\subsection{Self-diffusion}

Fig. 1 includes the prediction by the full PA scheme for the normalized short-time self-diffusion coefficient, $d_{S} / d_{0}$, of charged and neutral hard spheres, respectively, in comparison with ASD simulation results, and our DLS data for TPM coated charged silica spheres. The comparison of the full PA scheme result with the ASD data allows for deducing quantitatively the contribution to $d_{S}$ by the non-pairwise additive part of the HIs arising from the solvent-mediated interactions of three and more particles.

The large- $q$ regime related to self-diffusion is usually not accessible by DLS. Therefore, using an argument by Pusey (1978), we identify $d_{S}$ approximately as $d_{S} \approx D\left(q^{*}\right)$ (crosses in Fig. 1), where $q^{*}$ is the first wavenumber located to the right of $q_{m}$ where $S\left(q^{*}\right)=1$ (see top part of Fig. 3). Simulations of charged and neutral spheres have shown that $d_{S}$ is determined in this way to within $5-10 \%$ accuracy (Banchio \& Nägele, 2008; Abade et al., 2010). A comment is in order here on an incorrect proposition by Robert (2007), who claims that one of the present authors (G.N.) has pre-

IUCr macros version 2.1.1: 2009/05/11 
dicted theoretically that there is no difference in the concentration dependence of $d_{S}$ for charged and neutral particles. Quite the contrary, Nägele et al. have shown for charged spheres at low salinity that $d_{S} / d_{0} \approx 1-a_{t} \phi^{4 / 3}$ (cf. Eq. (6)), i.e., $d_{S}$ in these systems has a fractional $\phi$-dependence qualitatively different from that of hard spheres (Nägele et al., 1994; Nägele et al., 1995; Nägele, 1996). Only the self-diffusion coefficient of neutral hard spheres can be described by a regular virial series, with the first two virial coefficients given in Eq. (9). The correct second-order term, $-0.219 \phi^{2}$, in Eq. (9) differs even in its sign from the erroneous result, $+0.88 \phi^{2}$, used by Robert (2007).

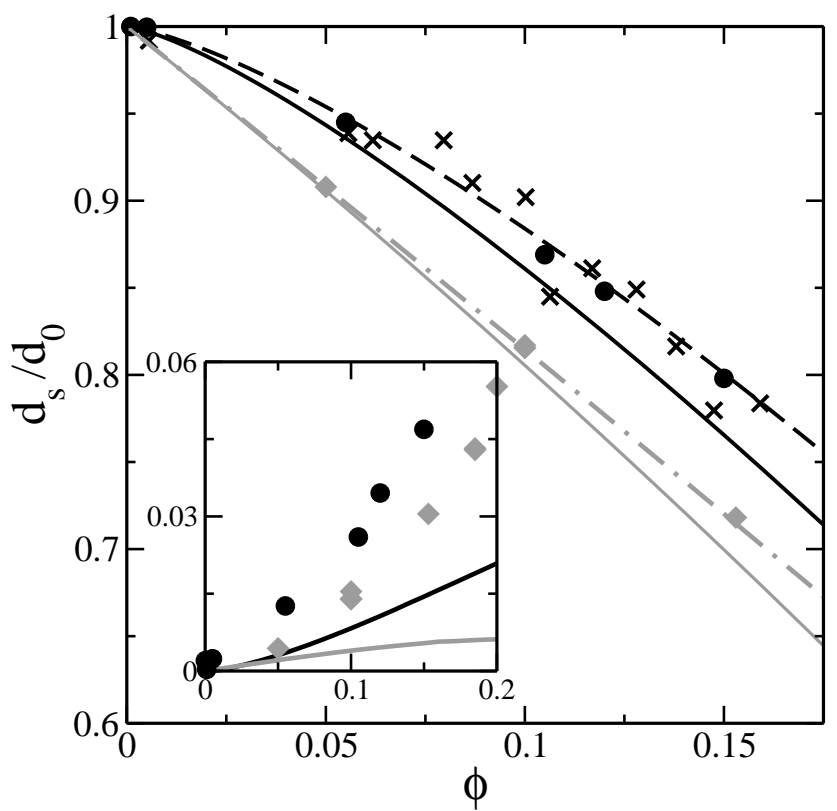

Fig. 1. Normalized short-time self-diffusion coefficient, $d_{S} / d_{0}$, of a deionized suspension of charged spheres (CS, black color) and hard spheres (HS, gray color), respectively. Crosses: DLS data for TPM coated charged silica spheres. Black circles and gray diamonds: accelerated Stokesian Dynamics (ASD) results for CS and HS, respectively. Solid black and gray lines: full PA-theory results for CS and HS, using the PBRMSA and Percus-Yevick input for $S(q)$, respectively. Dashed black line: $1-2.5 \phi^{4 / 3}$. Dashed-dotted gray line: 2 nd-order virial result for HS. Inset: $d_{S} / d_{0}$ as predicted by the full PA scheme (lines), and by ASD simulation (symbols), with the leading-order far-field HIs part for CS (black) and HS (gray), respectively, subtracted off. 
The $\phi^{4 / 3}$ dependence of $d_{S} / d_{0}-1$ was confirmed both by the experiments of Overbeck et al. (1999), and by ASD simulations based on the OMF model (Banchio \& Nägele, 2008). The depicted ASD data for a low salinity system of charged spheres are overall well described by the fractional $\phi$ relation in Eq. (6) for $a_{t} \approx 2.5$, over an extended range of volume fractions (black solid line in Fig. 1).

Considering the scatter in the charged silica spheres DLS data for $d_{S}$ depicted in Fig. 1, their overall $\phi$ dependence is consistent with the ASD simulation data, and the $\phi^{4 / 3}$ scaling prediction. The full PA scheme overestimates the strength of the HIs in non-dilute suspensions, for it does not account for the shielding of the HIs between a pair of particles by intervening other ones. This is the reason why, at intermediate and larger concentrations, the ASD and experimental data for $d_{S}$ are underestimated by the full PA scheme method. Note that the full PA scheme result for $d_{S} / d_{0}$ is still well described by the scaling relation in Eq. (6), but for a somewhat larger parameter value of $a_{t} \approx 2.9$. We emphasize here that hydrodynamic shielding is a many-body HIs effect which lowers the strength but not the range of the HIs. It should not be confused, as in earlier work (Riese et al., 2000), with the screening of the HIs by spatially fixed particles or boundaries that absorb momentum from the fluid, therefore causing a faster than $1 / r$ decay of the flow perturbation created by a point force (Diamant, 2007).

The hydrodynamic self-mobility related to $d_{S}$ is rather short-ranged, decaying like $1 / r^{4}$ for a large separation $r$ of two spheres. Consequently, the difference between $d_{S}$ and its infinite dilution value $d_{0}$ is smaller for charged spheres than for neutral ones, since electric repulsion disfavors near-contact configurations. The inset in Fig. 1 shows $d_{S} / d_{0}$, as obtained by the full PA scheme and ASD simulations, respectively, but with the far-field part originating from the leading-order self-mobility part proportional to $1 / r^{4}$ subtracted off. According to the inset, $d_{S}$ is rather insensitive to the near-field 
two-body part of the HIs, causing a small increase in $d_{S}$ only. The full PA scheme reproduces exactly the first-order virial coefficient, -1.832 , of hard spheres given in Eq. (9). This demonstrates the high precision of the numerical tables for the hydrodynamic pair mobilities used in our full PA scheme calculations. Three-body and higher-order HIs contributions come into play for $\phi \gtrsim 0.08$, with an enlarging influence on $d_{S}$ originating from hydrodynamic shielding.

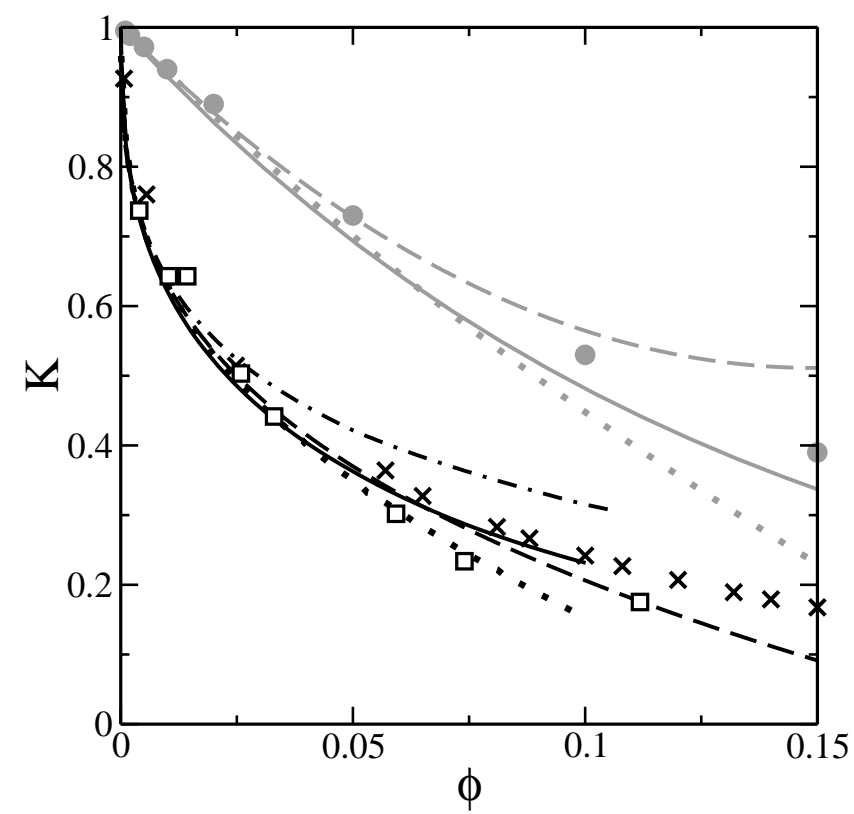

Fig. 2. Sedimentation coefficient, $K=U_{s} / U_{0}$, of charged spheres at low salinity compared with that of neutral hard spheres. Experimental DLS/SLS data are shown for charged polystyrene spheres in an ethanol-water mixture taken from Rojas-Ochoa (2004) (open squares), and for our TPM coated silica spheres (crosses). Solid (dotted) black lines: $\delta \gamma$ theory (full PA scheme) results, respectively, for the low-salinity polystyrene spheres system, obtained using the PBRMSA input for $S(q)$ with a fixed charge number $Z=200$. Dashed-dotted black line: $d_{S}$-corrected $\delta \gamma$ scheme result. Dashed black line: scaling form $1-1.71 \phi^{1 / 3}$ according to Eq. (5). Gray filled circles: hard-sphere simulation results by Ladd (1990). Dashed gray line: 2nd-order virial result for HS given in Eq. (8). Solid (dotted) gray lines: $\delta \gamma$ scheme (full PA scheme) results, respectively, for HS with Percus-Yevick input for $S(q)$. 


\subsection{Sedimentation}

In principle, one needs to distinguish between the short-time and the long-time sedimentation coefficients, but the latter is smaller than the first one by at most a few percent. The two coefficients are practically equal in dilute systems where the two-body HIs part dominates.

Fig. 2 includes theoretical, simulation and experimental results for the (short-time) sedimentation coefficient, $K$, of homogeneous systems for charged and neutral spheres. The key message conveyed by this figure is the qualitative difference in the $\phi$ dependence of $K$ for charged and neutral particles. This difference is more pronounced than the one for the self-diffusion coefficient discussed earlier. Charged spheres sediment more slowly than uncharged ones since near-contact configurations are disfavored. Thus, stronger laminar friction takes place between the back-flowing solvent, and the solvent layers dragged along with the settling spheres because of the stick hydrodynamic boundary condition. The solvent back-flow is created by a pressure gradient directed towards the container bottom which balances the non-zero, buoyancycorrected total gravitational force on the spheres.

At smaller $\phi$ and low salinity, $K$ is well described by the non-linear expression, $1-a_{s} \phi^{1 / 3}$, in Eq. (5), with a coefficient $a_{s} \approx 1.6-1.8$ depending to some extent on the strength of the electrostatic pair interactions. The exponent $1 / 3$ arises from the 2-body far-field part of the HIs which dominates the near-field part for $\phi \lesssim 0.08$, and the scaling relation, $q_{m} \propto \phi^{1 / 3}$, valid in low-salinity systems for the wavenumber location of the structure factor peak (Banchio \& Nägele, 2008). As a consequence, the $\phi^{1 / 3}$ concentration dependence of $K$ is observed both for dilute fluid and crystalline systems of charged particles.

The experimental results by Rojas-Ochoa (2004) for the low-salinity sedimentation coefficient of a suspension of charged polystyrene spheres in an ethanol/water mixture 
$\left(a=58.7 \mathrm{~nm}, n_{s}=1 \mu \mathrm{M}, L_{B}=1.48 \mathrm{~nm}\right)$, and also our data for the charged-silica spheres system, are in accord with the OMF-based prediction of a steep (as compared to neutral spheres) $\phi^{1 / 3}$-like decay of the sedimentation coefficient. The experimental values of $K$ in both systems discussed in Fig. 2 have been deduced from small- $q$ DLS and SLS measurements of $D(q)$ and $S(q)$, extrapolated to $q=0$. Therefore, there is an unavoidable scatter in the extrapolated data, in particular considering that the osmotic compressibility coefficient $S(0)$ of low-salinity systems is very small.

In contrast to charged particles, the small- $\phi$ dependence of $K$ is well represented for neutral spheres by a regular virial expansion. In fact, the second-order virial expression in Eq. (8) coincides, for $\phi \lesssim 0.08$, with the simulation data for $K^{\mathrm{HS}}$ by Ladd (1990). At larger $\phi$, shielding arising from the higher-order HIs terms comes into play, contributing to $K$ through the higher-order virial coefficients. Since shielding is disregarded in the full PA scheme, it notably overestimates the strength of the HIs for $\phi \gtrsim 0.1$. When applied to concentrations beyond its range of applicability, too small and eventually even non-physical negative values for $K$ are predicted (see the dotted lines in Fig. 2). The reason for the failure of the full PA scheme at higher $\phi$ is that it approximates the many-sphere hydrodynamic mobility matrix in a way that does not guarantee the positive definiteness of this matrix for all physically allowed particle configurations.

Fig. 2 displays additionally, both for neutral spheres and for the system parameters of the charged polystyrene spheres system (Rojas-Ochoa, 2004), the predictions for $K$ by the $\delta \gamma$ scheme of Beenakker \& Mazur (1984), and by the full PA scheme. The theoretical predictions for the silica system are not shown in order to not overburden the figure with too many curves.

For neutral hard spheres, in both analytic methods the Percus-Yevick solution for $S(q)$ is used as input. To leading order in $\phi$, we have obtained numerically that $K_{\mathrm{PA}}^{\mathrm{HS}}=$ 
$1-6.546 \phi$, in full agreement with the first-order coefficient in Eq. (8). For comparison, $K_{\delta \gamma}^{\mathrm{HS}}=1-7.339 \phi$. Thus, the $\delta \gamma$ scheme underestimate somewhat the hard-sphere sedimentation coefficient at small $\phi$. It is less accurate than the PA scheme at small $\phi$ owing to its incomplete account of two-body HIs contributions, notably its neglect of lubrication which plays a role for neutral spheres. Lubrication occurs in the thin fluid layer between two almost touching spheres in a relative squeezing or shearing motion. It is more influential to self-diffusion than to sedimentation, since in the latter case the (monodisperse) spheres move with equal mean velocities in the direction of the applied force field. In self-diffusion, on the other hand, a tagged particle is thermodynamically driven in a squeezing motion towards particles in front of it. At larger $\phi$, however, the $\delta \gamma$ scheme prediction for $K^{\mathrm{HS}}$ is closer to the simulation data than the full PA scheme result. We attribute this to the approximate inclusion of many-body HIs into the $\delta \gamma$ scheme which describe hydrodynamic shielding.

For charged spheres and small $\phi$, the full PA scheme result for $K$ follows precisely the scaling prediction $1-a_{s} \phi^{1 / 3}$, with $a_{s}=1.71$. The (uncorrected) $\delta \gamma$ scheme captures the overall $\phi$ dependence of $K$ at least in a qualitative way, both regarding the two considered charge-stabilized systems, and neutral hard spheres. Extensive comparisons with lower- $q$ ASD simulation data of $H(q)$, with Fig. 4 providing a representative example, show that the $\delta \gamma$ scheme has the tendency to somewhat underestimate $K$. The self-part corrected $\delta \gamma$ scheme, on the other hand, overestimates $K \approx H\left(q \ll q_{m}\right)$. At larger $q$ values, however, including the principal peak region of $H(q)$, it is in distinctly better agreement with the simulation data for $H(q)$ than the uncorrected version (Banchio \& Nägele, 2008). Indeed, the self-part corrected $\delta \gamma$ scheme result in Fig. 2 for charged polystyrene spheres, with the $d_{S}$ input calculated using the full PA scheme, lies distinctly above the experimental data for $K$. For the parameters of the polystyrene system, a least-square fit of the calculated sedimentation coefficients in 
the range $\phi \leq 0.02$ leads to Eq. (5), with coefficients $a_{s}=1.65,1.75$ and 1.71 for the $d_{S}$-corrected and uncorrected $\delta \gamma$ schemes, and the full PA scheme, respectively. In accord with the general trends discussed above, the full PA scheme result for $K$, which becomes exact al low $\phi$, is bracketed by the self-part corrected and uncorrected $\delta \gamma$ schemes predictions.

\subsection{Diffusion function}

We proceed by discussing the short-time diffusion function, $D(q)$, defined in Eq. (2), which is measured in short-time DLS and XPCS experiments. DLS data of its inverse, $d_{0} / D(q)$, are included in the bottom part of Fig. 3, for a low-salinity system of charged silica spheres at a volume fraction $\phi=0.15$ rather close to the freezing transition value. The experimental data are compared with our ASD simulation result for $D(q)$, the $\left(d_{S}\right.$-corrected) $\delta \gamma$ scheme result where the $d_{S}$-part is taken from the ASD simulation, and the full PA scheme prediction. For the two analytic schemes, the PBRMSA input for $S(q)$ shown in the top part of Fig. 3 was used.

The shape of $d_{0} / D(q)$ is similar to that of $S(q)$, owing to $d_{0} / D(q)=S(q) / H(q)$ according to Eq. (2). The analytic PBRMSA scheme predicts a structure factor in excellent agreement with our MC simulation data, and with the $S(q)$ obtained from the numerically elaborate RY scheme. The excellent agreement between all $S(q)$ depicted in the top part of Fig. 3, for all displayed wavenumbers, points to the accuracy of our scattering data. The only adjustable parameter in calculating $S(q)$ has been the effective charge number, uniquely determined as $Z=190$ by the PBRMSA, RY and MC methods, from matching the experimental $S\left(q_{m}\right)$.

IUCr macros version 2.1.1: 2009/05/11 


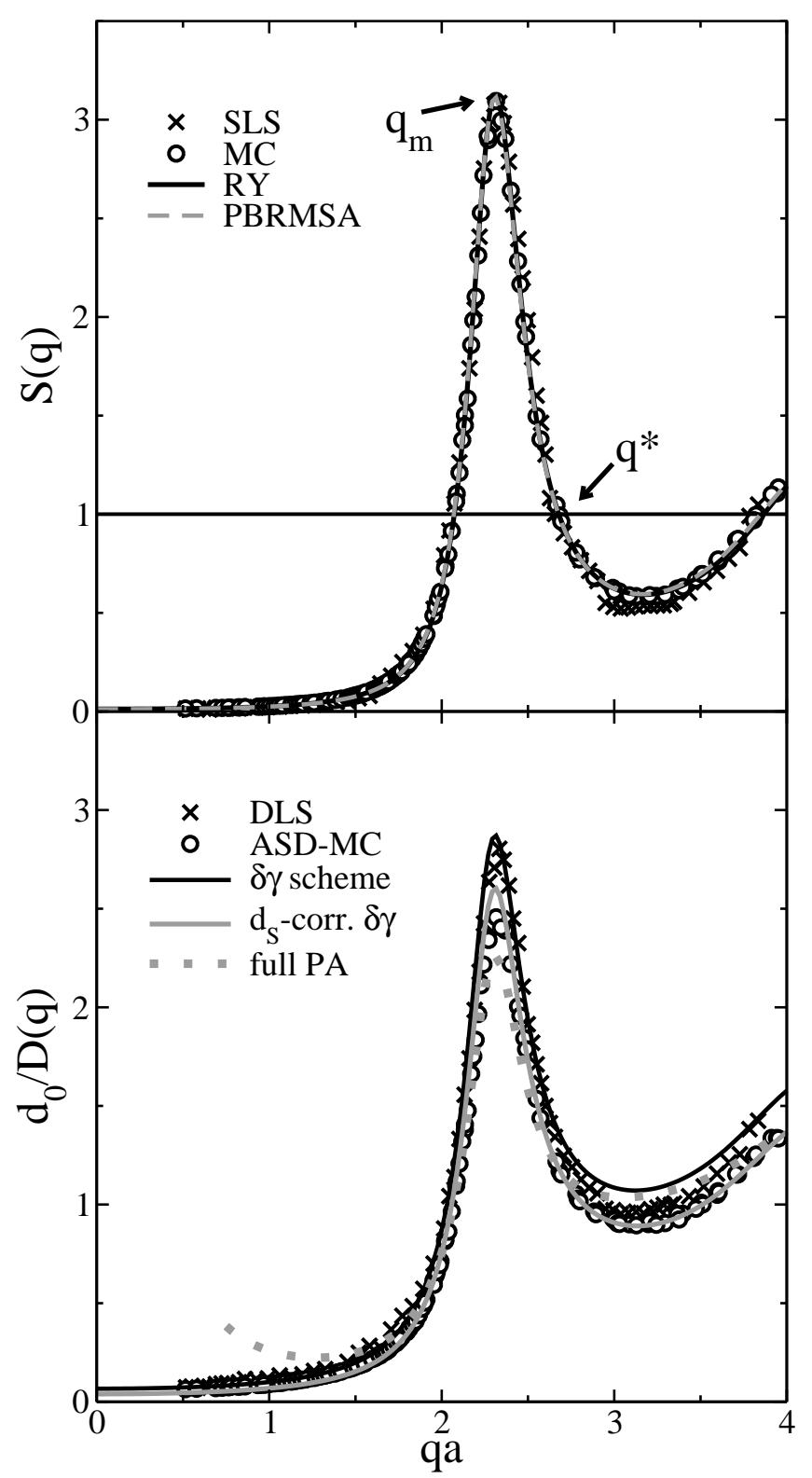

Fig. 3. Top: static structure factor, $S(q)$, obtained by static light scattering (SLS, crosses) and compared with PBRMSA and RY results (lines), and MC simulation data (open circles) for a common $Z=190$. Bottom: short-time inverse diffusion function, $d_{0} / D(q)$, for a low-salinity system of charged silica spheres. Crosses: DLS data. Open circles: ASD-MC simulation data. Solid black and gray lines: uncorrected and $d_{S}$-corrected $\delta \gamma$ scheme predictions, respectively. Dotted gray line: full PA method result. The system parameters are: $a=136 \mathrm{~nm}, \phi=0.15, n_{s}=0.7 \mu \mathrm{M}$, and $L_{B}=8.64 \mathrm{~nm}$.

We recall from Eq. (2) that $D(q \rightarrow \infty)=d_{S}$ and $D(q \rightarrow 0)=d_{0} K / S(q \rightarrow 0)=d_{c}$. 
Here, $d_{c}$ is the short-time collective diffusion coefficient which quantifies the initial decay rate of long-wavelength thermal concentration fluctuations. The short-time $d_{c}$ is only slightly larger than its long-time counterpart, even when a concentrated system is considered. The relative osmotic compressibility coefficient, $S(q \rightarrow 0)$, in the considered low-salinity system is very small, so that $d_{c}$ is exceedingly larger than $d_{0}$, reflected in Fig. 3 in the low- $q$ values of $d_{0} / D(q)$ close to zero. The function $D(q)$ attains its minimum at $q_{m}$. The so-called cage diffusion coefficient, $D\left(q_{m}\right)$, characterizes the slow relaxation of density fluctuations of a wavelength $\sim 2 \pi / q_{m}$ matching the radius of the nearest-neighbor shell. With increasing concentration and pair interactions, the cage stiffens, i.e., it becomes more sharply structured, as reflected by a smaller $D\left(q_{m}\right)$.

According to the bottom part of Fig. 3, there is good agreement between the ASD simulation data for $d_{0} / D(q)$, and the $d_{S}$-corrected $\delta \gamma$ scheme prediction with its PBRMSA input. The ASD simulation peak height is somewhat overestimated by the uncorrected $\delta \gamma$ scheme which uses a too small value for the self-diffusion coefficient of charged spheres (see Fig. 1). For the charged silica particles system considered here, the experimental peak height of $d_{0} / D(q)$ happens to be somewhat closer to that of the uncorrected $\delta \gamma$ scheme. However, the first minimum of $d_{0} / D(q)$ to the right of $q_{m}$ is in better accord with the corrected $\delta \gamma$ scheme prediction.

To illustrate the failure of the full PA scheme for concentrations $\phi \gtrsim 0.1$ where many-body HIs are strong, we have included its prediction into Fig. 3. It deviates from the experimental and simulation data most strongly at $q \approx 0$ and near $q_{m}$, reflecting its overestimation of the HIs at the large volume fraction $\phi=0.15$, by giving a too small value for $K$, and a too large value for $H\left(q_{m}\right)$.

IUCr macros version 2.1.1: 2009/05/11 


\subsection{Hydrodynamic function}

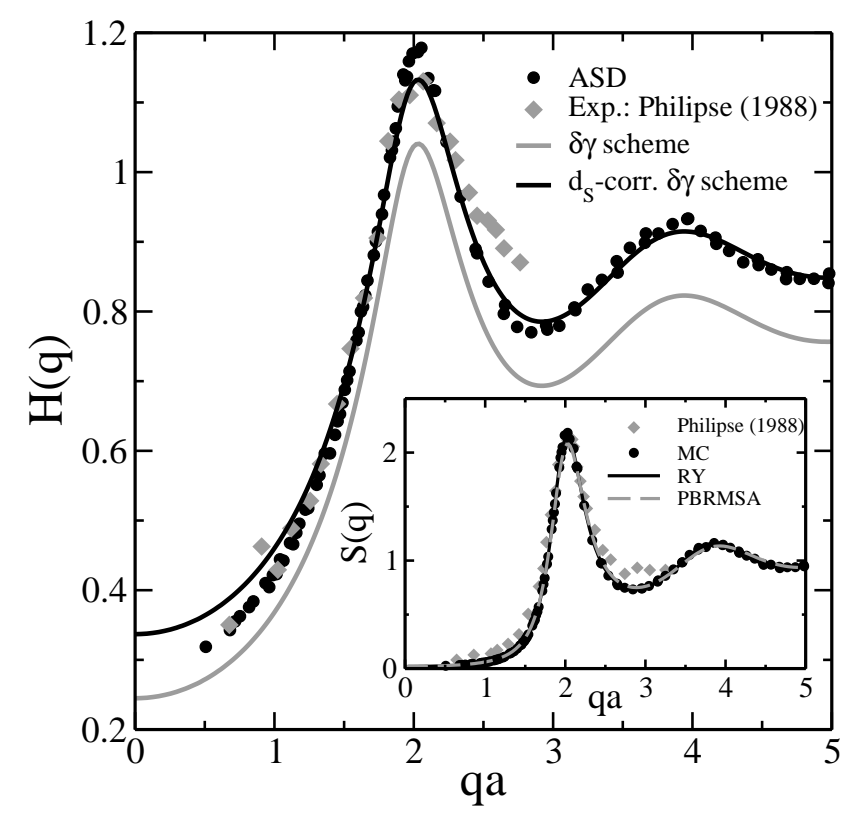

Fig. 4. Gray diamonds: DLS and SLS data for $H(q)$ and $S(q)$ (in inset), respectively, taken from Philipse \& Vrij (1988), for a charge-stabilized system at $\phi=0.101$, in comparison with corresponding ASD and MC data predictions (filled black circles). Solid gray and black lines: uncorrected and $d_{S}$-corrected $\delta \gamma$ scheme result, respectively. Black solid and dashed gray lines in inset: RY and PBRMSA $S(q)$, respectively, for $Z=100, a=80 \mathrm{~nm}, n_{s}=2 \mu \mathrm{M}$ and $L_{B}=5.62 \mathrm{~nm}$.

In Fig. 4 the experimental findings by Philipse \& Vrij (1988) for the $H(q)$ and $S(q)$ of a well-structured, charge-stabilized suspension of silica spheres suspended in a 70:30 toluene-ethanol mixture $(\epsilon=10$ at $T=298 \mathrm{~K})$, are compared with our theoretical and simulation predictions based on the OMF model. The PBRMSA, RY and MC $S(q)$ of common charge number $Z=100$ shown in the inset, coincide practically in the depicted $q$-range, demonstrating the accuracy of the PBRMSA scheme.

There is good overall agreement between the experimental $H(q)$, and the ASD and $d_{S^{-}}$-corrected $\delta \gamma$ scheme results (with $d_{S}$ taken from the ASD simulation), on accounting for the scatter in the experimental data for $H(q)$ which have been obtained from multiplying the experimental data for $D(q)$ by those for $S(q)$. The $d_{S}$-corrected IUCr macros version 2.1.1: 2009/05/11 
$\delta \gamma$ scheme underestimates to some extent the ASD $H\left(q_{m}\right)$, but except of the precise peak value the overall shape of $H(q)$ is well reproduced. Without $d_{S}$-correction, the ASD $H(q)$ is underestimated at all $q$, owing to the fact that the $\delta \gamma$ scheme predicts a too small value for the charged-particles $d_{S}$. Figs. 3 - 4 exemplify that the (self-part corrected) $\delta \gamma$ scheme allows for predicting consistently, and to almost quantitative accuracy, the short-time generic features of many charge-stabilized systems including small proteins and large colloidal spheres.

Low-salinity systems are typically characterized by a peak value of $H\left(q_{m}\right)$ larger than one. In a recent study based on the OMF model, the upper limiting freezing line for $H\left(q_{m}\right)$ was derived (Gapinski et al., 2010), from which it follows that $H\left(q_{m}\right)$ never exceeds the value of 1.3. However, $H\left(q_{m}\right)$ in low-salt systems is not always larger than one. At very low $\phi$, it increases monotonically according to $1+p_{m} \phi^{1 / 3}$, with a moderately system-dependent coefficient $p_{m}>0$ (see Eq. (7)). At larger $\phi$ where near-field HIs matter, $H\left(q_{m}\right)$ can pass through a maximum typically occurring at $\phi \sim 10^{-2}-10^{-1}$, with an ensuing decline when $\phi$ is further increased. Provided the system remains fluid at larger $\phi$, such as in apoferritin protein solutions (Gapinski et al., 2005), $H\left(q_{m}\right)$ can reach values smaller than one.

IUCr macros version 2.1.1: 2009/05/11 


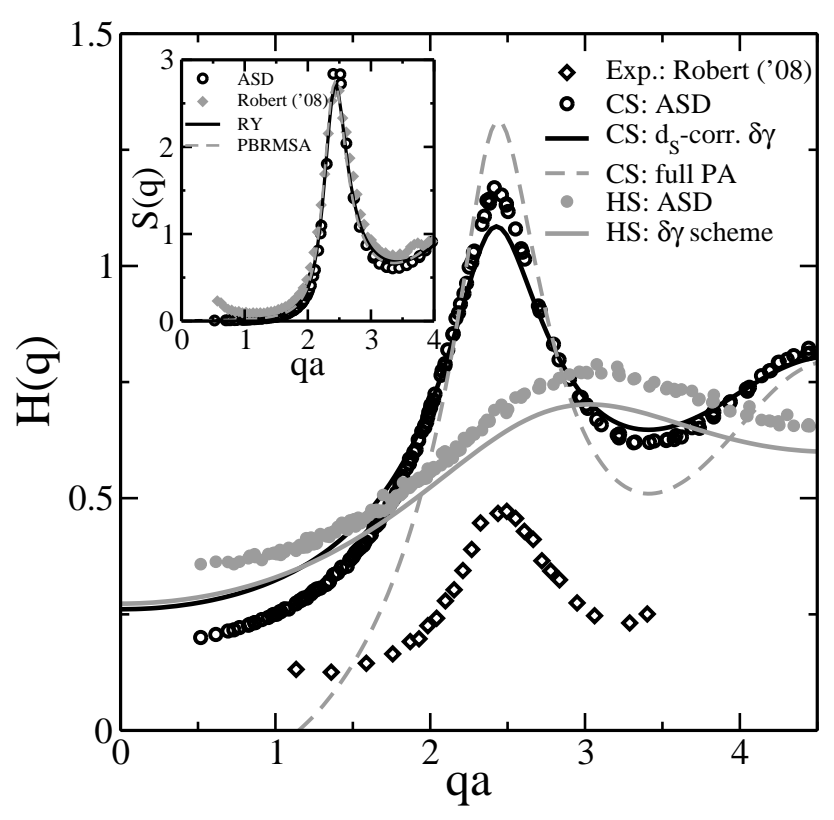

Fig. 5. XPCS and SAXS data of $H(q)$ and $S(q)$ (in inset), taken from Robert et al. (2008), for a low-salinity system of charged poly-perfluoropentylmethacrylate spheres (CS) with $a=62.5 \mathrm{~nm}, n_{s}=16 \mu \mathrm{M}$, and $\phi=0.18$ in a water/glycerol mixture at $T=293 \mathrm{~K}$ where $\epsilon=62.95$ and $L_{B}=0.91 \mathrm{~nm}$ (open diamonds). Inset: $\mathrm{RY}$ and PBRMSA $S(q)$ for $Z=163$. Comparison with OMF model based ASD data, $d_{S}$-corrected $\delta \gamma$ and full PA scheme results for $H(q)$, all obtained using $Z=163$. The experimental $H(q)$ is substantially smaller than the ASD $H^{\mathrm{HS}}(q)$ (gray circles), and the $\delta \gamma$ scheme result for hard spheres (gray solid line).

In the OMF model, $H\left(q_{m}\right)$ is bound from below by the corresponding peak height of neutral spheres. The latter decreases linearly in $\phi$ in the whole fluid phase regime (see Eq. (10)). At fixed $\phi$ and with increasing salt content, $H\left(q_{m}\right)$ and $d_{S}$ decrease monotonically, with $q_{m}$ shifted to larger $q$ values, towards the limiting hard-sphere values $H^{\mathrm{HS}}\left(q_{m}\right)$ and $d_{S}^{\mathrm{HS}}$, respectively. Opposite to this, $K$ increases monotonically with increasing salinity, for the reasons discussed earlier, towards its upper hard-sphere limit. In summary, the ordering relations

$$
\begin{aligned}
H\left(q_{m}\right) & \geq H^{\mathrm{HS}}\left(q_{m}\right) \\
d_{S} & \geq d_{S}^{\mathrm{HS}} \\
K & \leq K^{\mathrm{HS}},
\end{aligned}
$$


are fulfilled. The equality sign holds for zero particle charge, $Z=0$, and in the infinite salinity limit, $\kappa a \rightarrow \infty$. The OMF model ordering relations in Eq. (11) are obeyed by a large variety of experimentally studied charge-stabilized systems, including nanosized proteins (Gapinski et al., 2005), suspensions of compact colloidal particles (Philipse \& Vrij, 1988; Phalakornkul et al., 1996; Härtl et al., 1999; Rojas-Ochoa, 2004; RojasOchoa et al., 2003; Gapinski et al., 2009; Banchio et al., 2006; Holmqvist \& Nägele, 2010), and thermosensitive charged microgel spheres (Braibanti et al., in preparation).

In a series of articles, Robert, Grübel and coworkers (Grübel et al., 2008; Robert, 2007; Riese et al., 2000; Robert et al., 2008) reported on their observation of very small values for $H(q)$, for certain low-salinity suspensions of intermediately large volume fractions, which they studied by combining XPCS and SAXS techniques. At all probed wavenumbers, their $H(q)$ are substantially smaller than those of neutral hard spheres at the same $\phi$. This findings of so-called ultra-small $H(q)$ is incompatible with the OMF model since the first two ordering relations in Eq. (11) are violated.

A typical result for an ultra-small $H(q)$ with peak height $H\left(q_{m}\right) \approx 0.47$, taken from Robert et al. (2008) for a system of poly-perfluoropentylmethacrylate spheres in a water/glycerol mixture at $\phi=0.18$, is shown in Fig. 5 , in comparison with our OMF model based simulation and theoretical results for $H(q)$, which predict a peak value for $H(q)$ larger than one. The inset displays the experimentally determined $S(q)$, and the peak-height adjusted MC, RY and PBRMSA results obtained for the common charge value $Z=163$, and $n_{s}=16 \mu \mathrm{M}(\kappa a=1.46)$. The experimental peak height, $S\left(q_{m}\right) \approx 2.63$, identifies the system as fluid-ordered according to the Hansen-Verlet freezing rule. There is a visible small- $q$ upturn in the experimental $S(q)$ which is not reproduced by the OMF structure factors describing purely repulsive particles. However, this upturn should not be over-interpreted as a sign for a significantly influential particle attraction since the experimental $S(q)$ of very similar systems of charged poly- 
perfluoropentylmethacrylate spheres shown in Robert et al. (2008) do not have such an upturn. Moreover, the peak position of the experimental $S(q)$ in Fig. 5 fulfills the relation $q_{m}=1.1 \times 2 \pi n^{1 / 3}$ (Banchio \& Nägele, 2008), characteristic of a low-salinity system of electrostatically strongly repelling particles where van der Waals attraction plays no role. This follows also from standard DLVO theory when the parameters for the electric potential part quoted in the caption of Fig. 5 are used.

Just like in the silica system considered before, the $d_{S}$-corrected $\delta \gamma$ scheme result for $H(q)$, based on the PBRMSA $S(q)$ depicted in the inset input for its distinct part, and the precise ASD simulation result for its self-part, is in overall good agreement with the full ASD simulation result for $H(q)$. It underestimates the ASD peak height to some extent, but aside from the precise peak value the agreement with the ASD $H(q)$ is quantitatively good.

For completeness, Fig. 5 shows also the full PA scheme prediction for $H(q)$. The concentration here is clearly too large for the full PA scheme to apply, with the consequence that nonphysical negative values of $H(q)$ are predicted for $q a \lesssim 1.2$. This shows that for the present system, where $\phi=0.18, H(q)$ is strongly influenced by many-body HIs. Quite notably, however, for the same particle system, an ultra-small hydrodynamic function, with $H\left(q_{m}\right) \approx 0.7<H^{\mathrm{HS}}\left(q_{m}\right)=0.95$, was reported by Robert et al. (2008) even at $\phi=0.04$, i.e., for a concentration where two-body HIs dominate.

The experimental peak height in Fig. 5 is considerably smaller than the peak value, $H^{\mathrm{HS}}\left(q_{m}\right)=0.76$, of hard spheres, the latter calculated according to Eq. (10). To allow for a comparison at all probed $q$ values, Fig. 5 includes the ASD and $\delta \gamma$ scheme results for the $H(q)$ of hard spheres. The hard-sphere structure factor peak value is $S^{\mathrm{HS}}\left(q_{m}\right)=1.19$ at $\phi=0.18$.

Grübel, Robert and coworkers originally tried to explain their observation of strikingly low values for $H(q)$ as the result of HIs screening (Riese et al., 2000; Robert, 
2001). To support their assertion, they presented a Brinkman fluid-type calculation of $H(q)$ (Riese et al., 2000), wherein only the leading-order far-field part of the hydrodynamic pair mobility is considered, treating the Brinkman screening length as a fitting parameter. However, in a later experimental-theoretical study (Banchio et al., 2006), it was pointed out that hydrodynamic screening does not occur in fluid-ordered, unconfined suspensions of mobile colloidal particles (see here also Diamant (2007)). Furthermore, the assumed screening of the HIs conflicts with the fact that the short-time diffusion and viscosity properties of many charge-stabilized systems, at concentrations and interaction parameters similar to the ones probed by Robert et al., are well explained by OMF model based methods without any necessity to invoke HIs screening. The low-salinity system in Fig. 3, for example, is in the concentration range where an ultra-slow $H(q)$ should be observable.

More recently, Robert et al. retracted from their interpretation of ultra-small $H(q)$ 's as being due to HIs screening (Autenrieth et al., 2007). In an alternative attempt to explain their findings (Robert et al., 2008), they introduced a correction factor, $f=\eta_{0} / \eta_{\text {eff }}<1$, multiplying the OMF model based $\delta \gamma$ scheme $H(q)$, with the value of $f$ determined such that the ultra-small experimental $H(q)$ is overall matched. Furthermore, they conjecture that $f$ can be identified by the ratio of the solvent viscosity and some effective suspension viscosity $\eta_{\text {eff }}$, leaving it unspecified, however, whether $\eta_{\text {eff }}$ should be identified with the high-frequency viscosity, $\eta_{\infty}$, or with the substantially larger static suspension viscosity. This ad-hoc modification of the $\delta \gamma$ scheme lacks a sound physical basis, for the $\delta \gamma$ scheme expression for $H(q)$ describes a genuine diffusion property. The values for $f$ obtained from fitting the ultra-small $H(q)$ given by Robert et al. (2008), are neither consistent with calculated (Banchio \& Nägele, 2008) nor experimental (Bergenholtz et al., 1998) results for $\eta_{0} / \eta_{\infty}$. In this context, we note that the generalized Stokes-Einstein relation, $D\left(q_{m}\right) / d_{0} \times \eta_{\infty} / \eta_{0} \approx 1$, relating the cage 
diffusion coefficient to the short-time (high-frequency) viscosity, is valid approximately for neutral spheres only (Banchio \& Nägele, 2008; Abade et al., submitted), but not for low-salinity suspensions of charge-stabilized particles (Koenderink et al., 2003; Banchio \& Nägele, 2008).

\subsection{Influence of additional interactions}

In the following, we analyze the effect on $H(q)$ caused by particle interaction contributions not considered in the OMF model. On a qualitative level, we discuss the influence of particle porosity, residual attractive forces and microion kinetics on the shape of $H(q)$.

The effect of particle porosity on the $H(q)$ of dense suspensions of neutral porous spheres has been explored in a recent simulation study (Abade et al., 2010). A non-zero solvent permeability has the effect to weaken the HIs, reducing thus the deviations of $H(q)$ at all $q$ from its zero-concentration limiting value of one. For the same reason, a suspension of porous particles is less viscous than a suspension of impermeable ones (Abade et al., submitted). Porosity is less influential when the particles are charged since near-contact configurations are then unlikely. The particles studied by Robert et al. are only very weakly porous, if at all. Thus, porosity can not explain the findings of ultra-small $H(q)$ 's. On the contrary, significant porosity would lead to a $H(q)$ overall closer to one.

An attractive interaction contribution enlarges both $S(0)$ and the sedimentation coefficient $K$ (Moncha-Jordá et al., 2010). The enlargement of the latter is overcompensated by the former, at least at smaller $\phi$ (van den Broeck et al., 1981). Thus, in dispersions of moderately charged particles such as bovine serum albumin or lysozyme proteins with sufficiently strong short-range attraction, the collective diffusion coefficient, $d_{c}=d_{0} K / S(0)$, can attain values smaller than $d_{0}$ (Cichocki \& Felder-

IUCr macros version 2.1.1: 2009/05/11 
hof, 1990; Bowen \& Mongruel, 1998; Bowen et al., 2000; Prinsen \& Odijk, 2007).

Opposite to sedimentation, attraction tends to slow self-diffusion, resulting in smaller values of the short-time and long-time self-diffusion coefficients (Cichocki \& Felderhof, 1990; Seefeldt \& Solomon, 2003). Attraction-induced slowing of self-diffusion is accompanied by an augmentation of the short-time (high-frequency) and long-time (static) suspension viscosities (Woutersen et al., 1994). Attraction fosters the formation of short-lived, transient particle pairs and clusters, which are better shielded from the solvent backflow so that sedimentation is enhanced. In self-diffusion, however, the mean velocity of a weakly forced particle driven towards its next-neighbor cage particles becomes smaller with increasing attraction, owing to the larger tendency of nearby particles to form a transient cluster. This picture also explains why attraction-induced slowing of long-time self-diffusion is found not only in colloidal systems, where HIs are present, but also in atomic liquids (Bembenek \& Szamel, 2000). Sedimentation is different in the sense that all particles, not just a single tagged one, are forced to move, on the average, in the direction of the external force. Summarizing, the overall effect of attraction is to lower the difference between $H(\infty)-H(0)$, and to shift the peak position $q_{m}$ to larger values.

The data by Robert et al. for $S(q)$ and $H(q)$ give no hint on an appreciable attractive interaction part. The short-range van der Waals attraction acting between the particles is masked in low-salinity systems by the strong and long-ranged electric forces to such an extent that it can not influence $H(q)$ significantly. Moreover, the experimental $S(q)$ given by Robert et al. can be described to good accuracy by the OMF model based structure factor. Significant attraction would enlarge at low $q$ the gap between the OMF model and the ultra-small $H(q)$ in Fig. 5, instead of reducing it.

On first sight, the non-instantaneous electrokinetic relaxation of counter- and coions forming overlapping electric double layers around the charged colloids is a more IUCr macros version 2.1.1: 2009/05/11 
promising candidate for causing ultra-small $H(q)$ 's. Indeed, the relaxation of the microion clouds has a slowing influence on colloid diffusion, referred to as the electrolyte friction effect. This effect can lower $H(0)$ (Gapinski et al., 2005) to a smaller extent, and also the values of the short-time and long-time self-diffusion coefficients (McPhie \& Nägele, 2007). However, electrolyte friction scales with the ratio, $d_{0} / d_{m}$, of the free diffusion coefficient, $d_{0}$, of the slowly moving colloids relative to the (mean) free diffusion coefficient, $d_{m}$, of the small microions (Retailleau et al., 1999; Gapinski et al., 2005; McPhie \& Nägele, 2007). Because of the huge difference in these two free diffusion coefficients, it is unlikely that electrokinetics can explain the strikingly low values for $H(q)$ reported on by Robert and collaborators. Whereas the electrokinetic influence on colloid diffusion is very small for larger colloidal spheres, it can be significantly strong for small, nanosized macroions such as proteins.

\section{Conclusions}

The generic behavior of short-time diffusion properties in suspensions of chargestabilized colloidal particles with strong electrostatic interactions has been studied by simulation and analytic theory, in conjunction with dynamic light scattering on charged silica spheres. Our calculations are based on the OMF model which interpolates between the limiting cases of a deionized (low-salinity) system and a system of neutral hard spheres. Two analytic methods to determine $H(q)$, namely the full PA scheme and the (self-part corrected) $\delta \gamma$ scheme have been tested against Stokesian dynamics simulations and compared to experimental results. The full PA scheme becomes exact at very low volume fractions but it can not be applied to denser systems. The self-part corrected $\delta \gamma$ scheme gives overall good results in the whole liquid phase regime, for all wavenumbers except those in the low- $q$ regime. The experimental confirmation of OMF model based low- $\phi$ predictions, notably the $\phi^{1 / 3}$ scaling of $K$ (Rojas- 
Ochoa, 2004) and $H\left(q_{m}\right)$ (Härtl et al., 1999), the $\phi^{4 / 3}$ scaling of $d_{S} / d_{0}$ (Overbeck et al., 1999; Holmqvist \& Nägele, 2010), and in addition the $d_{S}^{r} / d_{0}^{r}=1-a_{r} \phi^{2}$ scaling (with $a_{r} \approx 1.3$ ) of the short-time rotational diffusion coefficient $d_{S}^{r}$, normalized by its zero-concentration limiting value $d_{0}^{r}$ (Koenderink et al., 2003), add to the credibility of the OMF model.

A large body of experimental results for $D(q)$ and $H(q)$, for systems of different particle types and sizes, concentrations, salt contents and solvents, is well described by the OMF model, with all the ordering relations in Eq. (11) satisfied. Residual attractive pair interactions or particle porosity, and most likely also electrolyte friction, can not explain the ultra-small $H(q)$ findings of Robert and collaborators. Ultra-small values of $H(q)$ have not been observed in our scattering experiments, nor in the ones by our collaborators and various other groups (Philipse \& Vrij, 1988; Phalakornkul et al., 1996; Rojas-Ochoa et al., 2003; Rojas-Ochoa, 2004).

Information on short-time dynamic properties is indispensable for a better understanding of long-time dynamic properties such as the static viscosity and the long-time self-diffusion coefficient. Short-time transport coefficients are used, e.g., as input in mode-coupling and dynamic density functional theory calculations of long-time properties. For charge-stabilized systems, we have shown recently (Holmqvist \& Nägele, 2010) that $d_{S}$ and $D(q)$ are linked to their corresponding long-time quantities by a simple, approximate scaling relation.

M.H. acknowledges support by the International Helmholtz Research School of Biophysics and Soft Matter (IHRS BioSoft). A.J.B. acknowledges financial support from SeCyT-UNC and CONICET. We thank J. Gapinski and A. Patkowski for helpful discussions. This work was under appropriation of funds from the Deutsche Forschungsgemeinschaft (SFB-TR6, project B2).

\section{References}

IUCr macros version 2.1.1: 2009/05/11 
Abade, G., Cichocki, B., Ekiel-Jezewska, M., Nägele, G. \& Wajnryb, E., (2010). J. Chem. Phys., 132, 014503; ibid. Phys. Rev. E, 81, 020401(R).

Abade, G., Cichocki, B., Ekiel-Jezewska, M., Nägele, G. \& Wajnryb, E. (submitted).

Autenrieth, T., Robert, A., Wagner, J. \& Grübel, G. (2007). J. Appl. Cryst. 40, 250.

Banchio, A. \& Brady, J. (2003). J. Chem. Phys. 118, 10323.

Banchio, A., Gapinski, J., Patkowski, A., Häussler, W., Fluerasu, A., Saccana, S., Holmqvist, P., Meier, G., Lettinga, M. \& Nägele, G. (2006). Phys. Rev. Lett. 96, 138303.

Banchio, A., McPhie, M. \& Nägele, G. (2008). J. Phys.: Cond. Mat. 20, 404213.

Banchio, A. \& Nägele, G. (2008). J. Chem. Phys. 128, 104903.

Banchio, A., Nägele, G. \& Bergenholtz, J. (1999). J. Chem. Phys. 111, 8721.

Beenakker, C. \& Mazur, P. (1984). Physica 126, p. 349.

Bembenek, S. \& Szamel, G. (2000). J. Phys. Chem. B, 104, 10647.

Bergenholtz, J., Horn, F., Richtering, W., Willenbacher, N. \& Wagner, N. (1998). Phys. Rev. $E, \mathbf{5 8}, \mathrm{R} 4088$.

Bowen, W., Liang, Y. \& Williams, P. (2000). Chem. Eng. Sci. 55, 2359.

Bowen, W. \& Mongruel, A. (1998). Colloids and Surfaces A, 138, 161.

Braibanti, M., Heinen, M., Haro-Perez, C., Rojas-Ochoa, L., Nägele, G. \& Trappe, V. (in preparation).

van den Broeck, C., Lostak, F. \& Lekkerkerker, H. (1981). J. Chem. Phys. 74, 2006.

Cichocki, B., Ekiel-Jezewska, M., Szymczak, P. \& Wajnryb, E. (2002). J. Chem. Phys. 117, 1231.

Cichocki, B., Ekiel-Jezewska, M. \& Wajnryb, E. (1999). J. Chem. Phys. 111, 3265.

Cichocki, B. \& Felderhof, B. (1990). J. Chem. Phys. 93, 4427.

Diamant, H. (2007). Israel Journal of Chemistry, 47, 225.

Gapinski, J., Patkowski, A., Banchio, A., Buitenhuis, J., Holmqvist, P., Lettinga, M., Meier, G. \& Nägele, G. (2009). J. Chem. Phys. 130, 084503.

Gapinski, J., Patkowski, A., Banchio, A., Holmqvist, P., Meier, G., Lettinga, M. \& Nägele, G. (2007). J. Chem. Phys. 126, 104905.

Gapinski, J., Patkowski, A. \& Nägele, G. (2010). J. Chem. Phys. 132, 054510.

Gapinski, J., Wilk, A., Patkowski, A., Häussler, W., Banchio, A., Pecora, R. \& Nägele, G. (2005). J. Chem. Phys. 123, 054708.

Grübel, G., Madsen, A. \& Robert, A. (2008). X-ray Photon Correlation Spectrocsopy (XPCS) in Soft-matter Characterization. Berlin: Springer-Verlag.

Härtl, W., Beck, C. \& Hempelmann, R. (1999). J. Chem. Phys. 110, 7070.

Heinen, M., Banchio, A. \& Nägele, G. (to be submitted).

Holmqvist, P. \& Nägele, G. (2010). Phys. Rev. Lett. 104, 058301.

Jeffrey, D. \& Onishi, Y. (1984). J. Fluid Mech. 139, 261.

Jones, R. \& Schmitz, R. (1988). Physica 149, p. 373.

Kim, S. \& Mifflin, R. (1985). Phys. Fluids, 28, 2033.

Koenderink, G., Zhang, H., Aarts, D., Lettinga, M., Philipse, A. \& Nägele, G. (2003). Faraday Discuss. 123, 335.

Ladd, A. (1990). J. Chem. Phys. 93, 3484.

McPhie, M. \& Nägele, G. (2007). J. Chem. Phys. 127, 034906.

Moncha-Jordá, A., Louis, A. \& Padding, J. (2010). Phys. Rev. Lett. 104, 068301.

Nägele, G. (1996). Physics Reports, 272(5-6), 215 - 372.

Nägele, G., Mandl, B. \& Klein, R. (1995). Progr. Colloid. Polym. Sci. 98, 117.

Nägele, G., Steininger, B., Genz, U. \& Klein, R. (1994). Physica Scripta T, 55, 119.

Overbeck, E., Sinn, C. \& Watzlawek, M. (1999). Phys. Rev. E, 60, 1936.

IUCr macros version 2.1.1: 2009/05/11 
Phalakornkul, J., Gast, A., Pecora, R., Nägele, G., Ferrante, A., Mandl-Steininger, B. \& Klein, R. (1996). Phys. Rev. E, 54, 661.

Philipse, A. P. \& Vrij, A. (1988). J. Chem. Phys, 88, 6459.

Prinsen, P. \& Odijk, T. (2007). J. Chem. Phys. 127, 115102.

Pusey, P. (1978). J. Phys. A, 11, 119.

Pusey, P. (1991). Liquids, Freezing and the Glass Transition. Amsterdam: Elsevier.

Retailleau, P., Riès-Kautt, M., Ducruix, A., Belloni, L., Candau, S. \& Munch, J. (1999). Europhys. Lett. 46, 154.

Riese, D., Wegdam, G., Vos, W., Sprik, R., Fenistein, D., Bongaerts, J. \& Grübel, G. (2000). Phys. Rev. Lett. 85, 5460.

Robert, A. (2001). Dynamic behavior of charge-stabilized colloidal suspensions. Ph.D. thesis, Université Joseph Fourier, Grenoble, France.

Robert, A. (2007). J. Appl. Cryst. 40, 34.

Robert, A., Wagner, J., Härtl, W., Autenrieth, T. \& Grübel, G. (2008). Eur. Phys. J. E, 25, 77.

Rogers, F. \& Young, D. (1984). Phys. Rev. A, 30, 999.

Rojas-Ochoa, L. (2004). Structure, dynamics and photon diffusion in charge-stabilized colloidal suspensions. Ph.D. thesis, Université de Fribourg, Switzerland.

Rojas-Ochoa, L., Vavrin, R., Urban, C., Kohlbrecher, J., Stradner, A., Scheffold, F. \& Schurtenberger, P. (2003). Faraday Discuss, 123, 385.

Seefeldt, K. \& Solomon, M. (2003). Phys. Rev. E, 67, 050402.

Segrè, P. \& Pusey, P. (1996). Phys. Rev. Lett. 77, 771.

Snook, I. \& Hayter, J. (1992). Langmuir, 8, 2280.

Watzlawek, M. \& Nägele, G. (1999). J. Colloid Interface Sci. 124, 170.

Woutersen, A., Mellema, J., Blom, C. \& de Kruif, C. (1994). J. Chem. Phys. 101, 542.

\section{Synopsis}

Short-time diffusion in charge-stabilized colloidal dispersions is studied by theory, simulation and scattering experiments. Generic features in self-diffusion, cage-diffusion and sedimentation are explored. 